\title{
Survey of Indoor Positioning Measurements, Methods and Techniques
}

\author{
Siddhesh Doiphode \\ Department of Computer \\ Engineering \\ Shree L.R. Tiwari COE \\ Mumbai University
}

\author{
J.W. Bakal, PhD \\ Principal, \\ Shivajirao S. Jondhale College \\ of Engineering \\ Mumbai, India
}

\author{
Madhuri Gedam \\ Department of Computer \\ Engineering \\ Shree L.R.Tiwari COE \\ Mumbai University
}

\begin{abstract}
The continuous improvement in the development in the field of communication networks and mobile computing, location based service (LBS) has become very popular in recent years. For outdoor positioning service the Global Positioning Systems (GPS) are the earliest widely used modern systems. In GPS technology Satellite signals cannot penetrate in indoor environment since they are blocked by building obstructions thus satellite signal cannot provide good accuracy in indoor environments due to lack of LoS(Line Of Sight). On doing research it is being observed that in the near future, indoor positioning will gain more demand as people spend more than $70 \%$ of their lives in indoor environment. A large variety of technologies have been designed for dealing with the problem since the indoor environments are very difficult to track. The paper also provide brief description on various indoor wireless tracking measurements, methodologies and technologies. The paper illustrates the theoretical points, the main tools, and the most promising technologies for indoor tracking infrastructure.
\end{abstract}

\section{General Terms}

Location Based Services, Indoor Positioning System, Fingerprinting Method, Propagation model.

\section{Keywords}

Trilateration, Triangulation, Propagation Model.

\section{INTRODUCTION}

Due to widespread advances of devices and technologies Indoor real-time locating systems (RTLSs) have been gaining relevance. RTLSs is indoor tracking where objects, vehicles, or people are tracked within a building or enclosed infrastructure. In market various indoor positioning solutions have been proposed such as Cellular-network [1], Computer Vision [2], Ultrasound [3], Infrared Ray [4], Bluetooth technique [5] etc. The early RTLSs hat uses ultrasound to the most recent ultra-wideband (UWB) techniques which are some of the adhoc solutions for indoor tracking. All these systems are able to provide accurate results however, they rely on additional hardware or large-scale infrastructures which incurs more cost. Due to significant cost, energy consumption and specific environment range limitations, such system are hard to deploy.

The latest trend in addressing indoor tracking problem is to use standard, low cost and make use of existing available technologies to reduce the cost. The forefront player in this competition is the smart phone enabled indoor positioning system (IPS) [6]. IPS based on Wi-Fi fingerprint is one of the most exploited technique and have received considerable attention recently[7] .The technologies used in the system are heterogeneous which encompasses $\mathrm{Wi}-\mathrm{Fi}$, UWB, radio-frequency identification (RFID), Bluetooth, nearfield communication (NFC), Long-Term Evolution (LTE). The latest challenges in indoor tracking is not only to design specialized sensor but also to implement data fusion method that can exploit the available technologies.

The WLAN RSS measurement techniques are used in Propagation model and Fingerprinting which are one of the fundamental method .To estimate the location of a mobile user with the help of the received signal strength information the path loss of the signal is taken into account. Geometric Techniques like trilateration [8] and triangulation $[9,10]$ can be used to determine the coordinates of a user. When the accuracy requirement is not very high the propagation model approach is relatively simple and efficient . However, due to the noise of wireless signals and the interference of indoor obstructions such as multi-story floors, doors and walls accurately measuring the distance based on signal attenuation is still difficult [11].

In this survey we are dealing with the problem of indoor wireless tracking. We present the type of measurement, different models and technologies used in Indoor tracking system.

The remainder of this paper is organized as follows. Section 2 introduces the type of measurement and Section 3 describes the overview of the different methods. Section 4 presents different technologies of IPS followed by conclusion.

\section{TYPES OF MEASUREMENT}

This section present different type of measurements used for tracking purpose.

\subsection{RSS}

The RSS measurement mainly based on the principle that greater the distance between two nodes, the weaker the strength of the received signal. Wi-Fi based IPS is commonly based on this type of measurement. The mapping between measured RSS and the distance between the receiving and the transmitting node is measured with the mathematical model using EuDistance.

The Euclidean distance which can be calculated as shown in the below equation is used to measure the distance between an online RSS value and the offline training database RSS records[12]. The smallest distance is used to decide which offline fingerprint is selected to determine the location of the user.

$$
\text { EuDistance }=\sqrt{ } \sum_{1}^{n}\left(R S S i-R S S i^{\prime}\right)^{2}
$$


In the above equation $\mathrm{n}$ is the number of APs or Wi-Fi fixed nodes, $\mathrm{RSS}_{\mathrm{i}}$ is the $\mathrm{i}^{\text {th }}$ AP's signal strength received in the online phase and $\mathrm{RSS}_{\mathrm{i}}{ }^{\prime}$ is the average RSS value in the training database. The main advantage of RSS based approach when compared with other existing method is the presence of RSS measurement in nearly all wireless system.

\subsection{TOA}

Is the travel time of a radio signal from a single transmitter to the remotely based server or receiver.The distance ' $d$ ' between a pair of nodes can be obtained by using measurements of signal propagation delay or time of flight (TOF) $\tau p=d / c$, where $c$ is the speed of electromagnetic waves in air $\left(\mathrm{c}=3^{*} 10^{8} \mathrm{~m} / \mathrm{s}\right)$.In TOA process node A transmits a packet to node $\mathrm{B}$ which in turn replies by transmitting an acknowledgement packet to A after a known or measured response delay $T_{d}$ [13].After receiving the acknowledgment node A estimate the signal round-trip time $\tau \mathrm{RT}=2 \tau \mathrm{p}+\tau \mathrm{d}$, from which it can calculate the distance without the need of a common time reference.

\subsection{TDOA}

Is the measured time difference between departing from one and arriving at the other station. The use of TDOA do not rely on absolute distance computed between pair of nodes. There are two methods to compute TDOA. In the first method multiple signals are broadcast from synchronized anchor nodes and the mobile phone or node measures the TDOA. In the second method a reference signal is broadcast by mobile phones or nodes and is received by several fixed anchors. The prerequisite for each method is that the anchor nodes are tightly synchronized through a network. To calculate the location of mobile node two TDOA measurements and at least three anchors are needed [14].

\subsection{PDOA}

In Phase-difference-of-arrival technique a couple of continuous wave signals at frequencies $\mathrm{f} 1$ and $\mathrm{f} 2$ are transmitted. Measurement of the phase difference at the receiver end results to be proportional to the distance and inversely to the difference $\mathrm{f} 2-\mathrm{f} 1$. Because of the presence of extremely small signal bandwidth phase estimation error can be very small. This technique was originally introduced for distance estimation in radar systems [13].

\subsection{Proximity}

In Proximity a binary connection between the mobile node and the anchor fixed node is required. The simplest method to define the proximity is to design a circle with a radius $r_{0}$ It is one of the simplest method to provide the location. The key advantage of this method is that it does not require any additional hardware and more importantly the time synchronization among nodes.

\section{METHODS}

In the previous section we discussed about various measurement techniques used for indoor tracking. We also discussed the estimation ways for each method along with is advantages and its requirement. Here we present the different methods for Indoor Tracking which includes Fingerprinting Method. We also discussed about the other well-known methods.

\subsection{Fingerprinting Method}

The technique of Fingerprint positioning is received signal strength (RSS) based positioning that uses location dependent characteristics with a location and uses these characteristics to estimate the position. Fingerprint based indoor positioning consists of two phases named as offline and online phases. Fingerprint database is established in offline phase and positioning is performed in online phase [14,15]. RSS values that are obtained from access points on a specific time period at the predetermined reference points are stored in a database that is called as fingerprint database. Each fingerprint in this database contains basically location information and RSS values obtained from surrounding access points at that location.

The architecture of a WLAN RSS fingerprinting system in shown in Figure3.1.In the offline phase the RSS fingerprints are sampled at each reference position from a number of access points (AP). APs are usually fixed transmitter such as Wi-Fi routers. A reference position is the position in the indoor environment that needs to be tracked. And the signal fingerprint at each RP are updated or inserted in the survey database. The received signal strength (RSS) can be defined as the measure of signal power from an AP to a receiver which can be sampled without any additional requirement in WLAN environment. Single samples of RSSI recorded from the nearby access point are not sufficient to characterize a fingerprint. Due to presence of noise in the environment it is necessary to obtain an average of the readings to successfully identify a fingerprint. In the online phase the background service running on the users mobile measures a vector of RSS value at an unknown location and then compares the RSS value received in the online determination phase with the training database. With the help of positioning algorithm the most likely location of mobile user is finally calculated.

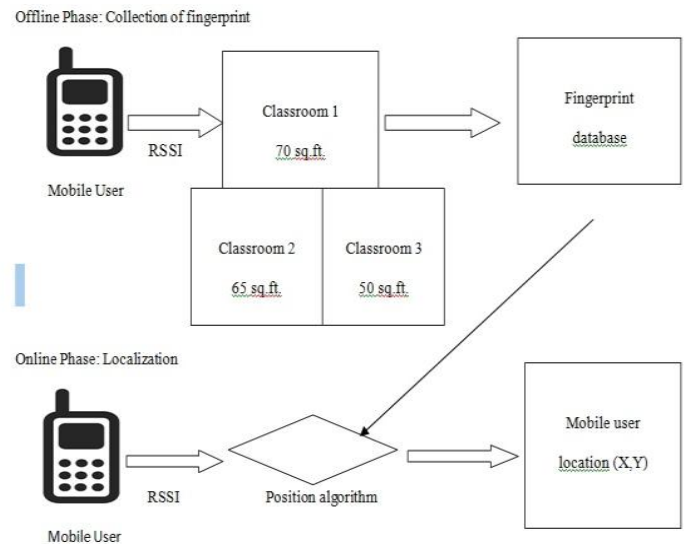

Figure 3.1 Fingerprint Method

\section{TECHNOLOGIES}

The number of technologies for Indoor Positioning is ever growing due to advance technologies which includes ShortRange Wireless Technologies, UWB Technology, Cellular Networks, and Near-Field Technology. This section gives brief description about the technologies.

\subsection{Short Range Wireless Technologies}

It includes various technologies like Wi-Fi for wireless local area network (WLAN), RFID and NFC for object identification, Bluetooth for wireless personal area network. The main intention for developing these technologies was not for location tracking purpose.

4.1.1 WLAN (IEEE 802.11)

The wireless local area network (WLAN) operating in 2.4 $\mathrm{GHz}$ has become very popular in public and enterprise area during last few years. Exploiting such opportunity to use an existing WLAN infrastructure for indoor location will thus 
improve the acceptance of the location service by most of them. Using RSS measurement technique in WLAN positioning systems the accuracy is approximately 3 to 30 $\mathrm{m}$, with an update rate of few seconds.

\subsubsection{Bluetooth}

The Bluetooth Low Energy (BLE) can be used for indoor positioning. This technology requires device supporting BLE which is the $4^{\text {th }}$ major revision of Bluetooth specification. In this technology a device which generates iBeacon advertisements is called beacon. Beacons establish a region around them by iBeacon signals. Beacons transmit advertisement data frames containing three identifying fields:

UUID: universally unique identifier is 128 bits integer, which can be used as an ID for all beacons used in an application

Major: major value is a 16 bits integer, which can be used to differentiate between beacons with same UUIDs.

Minor: this 16 bits integer can be used to differentiate between beacons with the same UUIDs and Major values. iBeacon regions are defined by these three identifying signals, so it introduces a flexible approach to positioning. Beacons can be attached to the movable objects which are not tied to a location[16].

\subsubsection{Cellular Networks}

To estimate the location of outdoor mobile clients Global system of mobile/code division multiple access (GSM/CDMA) mobile cellular network are used. However the same technology cannot be used for indoor tracking system to estimate the location of indoor mobile clients. Indoor positioning based on mobile cellular network is possible if the building is covered by several base stations or one base station with strong RSS received by indoor mobile clients. The author presented a GSM-based indoor localization system [18]. Their key idea that makes accurate GSM-based in-door localization possible is the use of wide signal-strength fingerprints. The higher the dimensionality of base station the better the accuracy for Indoor positioning System.5G mobile technology has gained more importance due to the adoption of small cells and massive antenna arrays at millimeter waves [19].

\subsubsection{UWB Technology}

This technology is growing interest since February 2002 for use by UWB devices [20]. In UWB Active RFID tag transmits UWB signals (unknown coordinates).A computer calculates the position of the tag with the time difference of arrival. UWB provides good accuracy due to the easy estimation of TOA with a fine resolution which translates the ranging estimation. Therefore UWB can be viewed as promising technology in Indoor positioning.
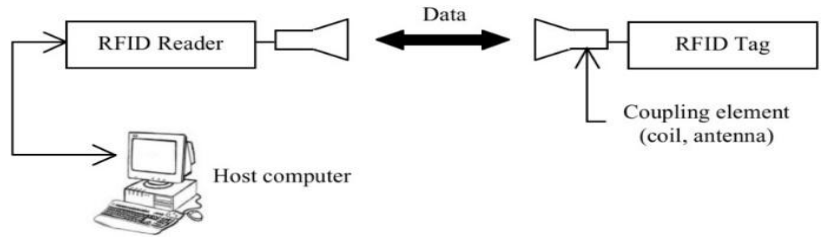

Figure 4.1 Localization of an Active RFID tag

\subsubsection{Near-Field Technology}

This technology is based on near field electromagnetic ranging (NFER) that uses low frequency upto $(1 \mathrm{MHz})$ and long wavelength $(300 \mathrm{~m})$.The main idea behind this technology is to take advantage of deterministic relationship that exists between angle formed by magnetic field and electric of the received signal and the distance between receiver and the transmitter. The main drawback is the use of large antennas used which leads to high cost.

The summary of the above mentioned technologies is compared on basis of different parameters like measurement techniques, accuracy. Along with that the pros and cons of the discussed technologies is mentioned.

Table 1: Comparison of Existing Positioning System

\begin{tabular}{|c|c|c|c|c|}
\hline Technology & $\begin{array}{l}\text { Measurement } \\
\text { Techniques }\end{array}$ & Accuracy & Pros & Cons \\
\hline GPS & TDOA & $10-20 \mathrm{~m}$ & Earth Coverage & $\begin{array}{l}\text { Only Outdoor Position, } \\
\text { Very Expensive }\end{array}$ \\
\hline Cellular $(2 \mathrm{G} / 3 \mathrm{G})$ & OTDOA & $50-500 \mathrm{~m}$ & Country Coverage & $\begin{array}{l}\text { Requires synchronized } \\
\text { base station }\end{array}$ \\
\hline Wi-Fi & RSS-Fingerprinting & $1-5 m$ & $\begin{array}{l}\text { Indoor coverage, } \\
\text { Low cost }\end{array}$ & $\begin{array}{l}\text { Large training database } \\
\text { required }\end{array}$ \\
\hline UWB & TOA/TDOA & $0.1-1 \mathrm{~m}$ & Indoor coverage & Short Range \\
\hline RFID/Bluetooth & Proximity & Connectivity Range & $\begin{array}{c}\text { Indoor coverage, low } \\
\text { cost }\end{array}$ & $\begin{array}{c}\text { Low accuracy, one tag } \\
\text { per location }\end{array}$ \\
\hline NFR & Electromagnetic filed & $1-5 \mathrm{~m}$ & Indoor coverage & Large antennas \\
\hline
\end{tabular}




\section{CONCLUSION}

This paper gives brief idea about current Indoor positioning system. In this paper we have discussed about various Indoor positioning technologies, models and also made the comparison for the same. We also reviewed the types of measurements that are used to track the said object. The emphasis is on the Wi-Fi Technology and the Fingerprint method. The existing system can also be integrated to get a hybrid solution which can serve various problem.

\section{REFERENCES}

[1] S.Tekinay, "Wireless geolocation systems and services,"IEEE Communications Magazine, vol. 36, no.4, pp. 28-29, April 1998Ding, W. and Marchionini, G. 1997 A Study on Video Browsing Strategies. Technical Report. University of Maryland at College Park.

[2] J. Krumm, S. Harris, B. Meyers, B. Brumitt, M. Hale, and S. Shafer, "Multi-camera multi-person tracking for easy living," in Proceedings of IEEE International Workshop on Visual Surveillance, pp. 3-10, Washington, DC, July 2000

[3] T. Gigl, G. J. M. Janssen, V. Dizdarevic, K.Witrisal, and Z. Irahhauten, "Analysis of a UWB Indoor Positioning System Based on Received SignalStrength," in Positioning, Navigation and Communication, 2007. WPNC '07. 4th Workshop, 2007, pp. 97-101

[4] R. Azuma, "Tracking requirements for augmented reality,"Communications of ACM, vol. 36, no. 7, pp. 5051, July 1993

[5] R. Azuma, "Tracking requirements for augmented reality,"Communications of ACM, vol. 36, no. 7, pp. 5051, July 1993

[6] Hadis Kakanejadi Fard, Yuanzhu Chen, and Kyung Kook Son "Indoor Positioning of Mobil Deviceswith Agile iBeacon Deployment"

[7] Chouchang Yang and Huai-Rong Shao"The Future of Wi-Fi WiFi-Based Indoor Positioning" IEEE Communications Magazine March 2015

[8] S. Mazuelas, A. Bahillo, R. Lorenzo, P.Fernandez, F.Lago, E. Garcia, J. Blas, and E. Abril, "Robust indoor positioning provided by real-time RSSI values in unmodified WLAN networks," IEEE Journal Selected Topics in Signal Processing, vol. 3,pp

[9] H. Liu, H. Darabi, P. Banerjee, and J. Liu, "Survey of wireless indoor positioning techniques and systems,"IEEE Transactions on Systems,Man, and Cybernetics, Part C, vol. 37, pp. 1067-1080,November 2007M.
[10] M. Robinson and I. Psaromiligkos, "Received signal strength based location estimation of a wireless LAN client," in Proceedings of IEEE Wireless Communications and Networking Conference, vol. 4,pp.2350-2354, New Orleans, LA, March 2005

[11] K. Curran, E. Furey, T. Lunney, J. Santos, D.Woods, and A. McCaughey, "An evaluation of Indoor location determination technologies," J.Location Based Services, vol. 5, no. 2, pp. 61-78, 2011

[12] J. M. Castro-Arvizu, P. Closas, and J. A. FernándezRubio, "Cramér-Rao lower bound for breakpoint Distance estimation in a path-loss model," in Proc.IEEE ICC Workshop Adv. Netw. Localization Navigation. Sydney, Australia, Jun. 2014.

[13] Z. Sahinoglu, S. Gezici, and I. Guvenc, Ultra-Wideband Positioning Systems: Theoretical Limits,Ranging Algorithms, and Protocols Cambridge, U.K.: Cambridge Univ. Press, 2008

[14] B. T. Fang, "Simple solutions for hyperbolic and Related position fixes,"IEEE Trans. Aerospace Electron. Syst., vol. 26, no. 5, pp. 748-753 Sep. 1990.

[15] M. Scherhaufl et al., "Indoor localization of passive UHF RFID tags based on phase-of-arrival evaluation,"IEEE Trans. M icrow. Theory Techn. vol. 61, no. 12, pp. 4724-4729, Dec. 2013.

[16] Sinem Bozkurt, Ahmet YazÕcÕ , Serkan Gunal, Ugur Yayan, Fatih Inan "A Novel Multi-Sensor And MultiTopological Database for Indoor Positioning on Fingerprint Techniques" IEEE

[17] W. Yeung and J. Ng, “An enhanced wireless LAN positioning algorithm based on the fingerprint Approach," in Proceedings of IEEE TENCON, pp.1-4, Hong Kong, November 2006

[18] V. Otsason, A. Varshavsky, A. LaMarca, and E. de Lara, "Accurate GSM indoor localization,"UbiComp 2005 Lecture Notes Computer Science, Springer-Varlag, vol. 3660, pp. 141-158, 2005.

[19] R. D. Taranto, R. Raulefs, D. Slock, T. Svensson, and H. Wymeer-sch, "Location-aware communications for 5G networks," IEEE Signal Process. Mag., vol. 31, no. 6, pp.102-112, Nov.2014.

[20] Fed. Commun. Comm., "Revision of Part 15 of the Commission's rules regarding ultra-wideband Transmission systems, first report and order," Washington, DC, USA, ET Docket 98-153, Feb. 14, 2002, Apr. 